\title{
TTR
}

Traduction, terminologie, re?daction

\section{Freud : N.d.T. ou Des affects et fantasmes chez les traducteurs de Freud}

\section{Ginette Michaud}

Volume 2, numéro 2, 2e semestre 1989

L'erreur en traduction

URI : https://id.erudit.org/iderudit/037049ar

DOI : https://doi.org/10.7202/037049ar

Aller au sommaire du numéro

Éditeur(s)

Association canadienne de traductologie

ISSN

0835-8443 (imprimé)

1708-2188 (numérique)

Découvrir la revue

Citer cet article

Michaud, G. (1989). Freud : N.d.T. ou Des affects et fantasmes chez les traducteurs de Freud. TTR, 2(2), 105-127. https://doi.org/10.7202/037049ar d'utilisation que vous pouvez consulter en ligne.

https://apropos.erudit.org/fr/usagers/politique-dutilisation/ 


\title{
Freud: N.d.T. ou Des affects et fantasmes chez les traducteurs de Freud
}

\author{
Ginette Michaud
}

«Our translations, even the best ones, proceed from a wrong premise. They want to turn Hindi, Greek, English into German instead of turning German into Hindi, Greek, English. Our translators have a far greater reverence for the usage of their own language than for the spirit of the foreign works [...]. The basic error of the translator is that he preserves the state in which his own language happens to be instead of allowing his language to be powerfully affected by the foreign tongue. [...] He must expand and deepen his language by means of the foreign language. It is not generally realized to what extent this is possible, to what extent any language can be transformed, how language differs from language almost the way dialect differs from dialect ; however, this last is true only if one takes language seriously enough, not if one takes it lightly. "

Rudolph Pannwitz, Die Krisis der europäischen Kultur (cité par

Walter Benjamin, dans «The Task of the Translator $»)$ 
Le titre du présent article* fait fond pour une part sur un texte récemment publié dans la revue Études françaises ${ }^{1}$ dans lequel j'amorçais une critique de la nouvelle traduction du volume XIII des $\mathscr{E}$ Euvres complètes de Freud. Psychanalyse ${ }^{2}-$ tel est en effet le titre retenu par l'équipe de Jean Laplanche et d'André Bourguignon. Les principes éditoriaux et le fameux glossaire ${ }^{3}$ des termes pré-traduits qui ont guidé leur entreprise n'avaient pas encore été publiés à l'époque où j'ai rédigé cette chronique : ils l'ont été depuis, mais je doute fort, après en avoir pris connaissance, que les raisons (et le mot résonne ici au plus près d'une ratio et d'une rationalisation du geste de traduire) avancées pour justifier leurs décisions modifient cette première réaction et parviennent à vraiment emporter l'adhésion du lecteur qui trouvera bien moins dans cette nouvelle traduction un Freud en français, hélas !, qu'un "français freudien ${ }^{4}$ ", c'est-à-dire une langue très curieuse et aussi peu courante (et coulante) que possible, un discours qui s'apparente moins à la transposition - transformation, expansion, approfondissement selon les termes de Pannwitz - inspirée d'une écriture, jusque dans son étrangeté, qu'à la restitution du sens du texte original par une sorte de jargon maniaque où, plus que jamais, la psychanalyse semble se replier, sous la figure idéalisée et surmoïque du scientisme régnant, sur une image totalement professionnelle et technocratique d'elle-même.

Je ne veux pas reprendre ici cette critique, qui abordait quelquesuns des problèmes de terminologie et de conceptualisation soulevés par cette traduction dite "scientifique" de Freud, qu'il s'agisse par exemple des diverses techniques adoptées pour rendre les doublets et les mots composés si fréquents en allemand, de la discutable pratique de néologisation poursuivie ici, où l'on cherche à «moderniser» le lexique freudien en ayant recours à des usages archaïques d'un français depuis longtemps tombé en désuétude ou oublié (par exemple : "désirance » pour «nostalgie», «frustrané " pour «vain», « désaide » pour

* Cet article est la version remaniée d'une communication présentée au colloque sur "La traduction de la psychanalyse / La psychanalyse comme traduction ", organisé par l'Association canadienne de littérature comparée et l'Association canadienne de traductologie, Congrès des sociétés savantes, Université Laval, le 30 mai 1989. Ce travail s'inscrit à l'intérieur d'une recherche plus vaste portant sur les figures du Sujet-Nation, subventionnée par le CRSH.

1. «Freud : N.d.T. ", Études françaises, 24 :3, 1988, pp. 125-140.

2. Sigmund Freud, Euvres complètes. Psychanalyse, volume XIII, 1914-1915, Paris, Presses universitaires de France, 1988, $350 \mathrm{p}$.

3. André Bourguignon, Pierre Cotet, Jean Laplanche et François Robert, Traduire Freud, Paris, Presses universitaires de France, 1989, 379 p.

4. Pierre Cotet, "Notre idée est de faire un français freudien", Libération, 14 avril 1988 , p. XVII. 
« détresse », " coulpe » pour « culpabilité ", " refusement " pour " frustration », etc.), ou encore, plus généralement, du parti-pris de littéralité compris dans son acception la plus étroite, qui entend respecter jusqu'à l'ordre même des mots dans la phrase en allemand, principe qui a déjà fait l'objet de multiples critiques lors des Cinquièmes Assises de la traduction littéraire qui ont eu lieu à Arles en novembre 1988. Sans négliger l'importance et les conséquences de ces choix qui, loin d'avoir seulement une portée technique, mériteraient chacun une discussion détaillée qui doit encore être réservée, je voudrais pour le moment plutôt infléchir mon titre, "N.d.T.", dans un autre sens, plus littéral et plus marginal peut-être, du moins à première vue, en l'orientant vers ces « Notes du traducteur » qui donnent à lire le transfert à l'œuvre dans la traduction, notamment dans quelques-uns des affects et des fantasmes des traducteurs qui semblent tout particulièrement avivés par la tâche de traduire Freud, si l'on en juge par la vigueur du débat qui marque la "crise de la traduction " depuis que l'on a "touché " à nouveau au texte freudien ${ }^{5}$. La «crise de vers» et «l'orage lustral " dont parlait Mallarmé paraissent presque faibles en comparaison, et la psychanalyste Élisabeth Roudinesco a raison de se dire horrifiée par la vulgarité des propos entendus, par la mesquinerie et l'intolérance des attaques qui fusent de part et d'autre: ainsi, «des néo-lacaniens de tendance millerienne», rapportait-elle récemment à propos d'un numéro de l'Âne consacré à cette question, seraient «allés jusqu'à manier l'insulte contre le clan de l'autre bord en accusant ladite équipe de s'être livrée sur le texte freudien à un travail semblable à celui des nazis qui dans les années trente avaient "aryanisé " le vocabulaire de la psychanalyse pour le rendre conforme à leur politique d'extermination antijuive ${ }^{6}$ ". On comprend sans peine que, devant la violence de tels propos, la polémique ait atteint son niveau le plus bas et qu'elle rende difficile, et partant d'autant plus nécessaire, tout effort de réflexion et de critique réelle que l'on pourrait adresser à cette nouvelle traduction, dont le défaut majeur, souligne Roudinesco, est «à la fois d'ignorer la conceptualité lacanienne et de céder à l'illusion de la transparence absolue ${ }^{7}$ ". Je reviendrai plus loin sur cette conception

5. Voir, entre autres, les comptes rendus d'Élisabeth Roudinesco (« Freud à vos souhaits ", Libération, 14 avril 1988), les feuilles publicitaires de l'équipe Laplanche (le Monde, 25 avril 1988 et 2 mai 1988), le débat autour de cette traduction (le Monde, "Le monde des livres", 15 avril 1988, 18 novembre $1988 ; l^{\prime} \hat{A n e}, \mathrm{n}^{\mathrm{os}} 35-36$ ), et tout particulièrement l'excellent article de Jacques Le Rider, "Freud et ses faux amis traducteurs", le Monde, "Le Monde des livres », 28 avril 1989 , p. 22.

6. Élisabeth Roudinesco, "Repli individuel et malaise collectif ", le Magazine littérai$r e, \mathrm{n}^{\circ} 264$, avril 1989 , p. 78 .

7. Ibid. 
de la traduction de l'équipe de Laplanche et sur le transfert au texte freudien qu'elle implique, mais je voudrais auparavant examiner ce qui joue, à mon avis de façon fondamentale, dans ce petit signe, "N.d.T. ", qui, le plus souvent refoulé en note en bas de page, marque toujours de façon significative un manque à traduire, une défaillance, voire une résistance du traducteur, et laisse en tout cas affleurer plus clairement qu'ailleurs son angoisse.

\section{Métaphores de la traduction}

De façon générale, on remarquera que cette convention fait le plus souvent l'objet d'un usage tacitement réglé chez les traducteurs. Ces «N.d.T. - qu'elles adoptent un style télégraphique ou celui, plus élaboré, de la glose - risquent en effet, si elles se multiplient, de paralyser toute décision chez le traducteur et elles devront donc, pour cette raison, être sévèrement contrôlées et si possible bannies des limites de l'opération traduisante : elles sont donc renvoyées, et souvent non sans mépris, du côté du commentaire et de l'explication, activités interprétatives perçues comme totalement incompatibles avec "les principes d'économie significative qui doivent dominer une traduction", selon l'expression de Lacan $^{8}$, économie de la traduction toujours serrée, mesurée, du "less is more». Or, il faut se demander si le contact d'un texte qui, comme celui du fondateur de la psychanalyse, peut être à plusieurs égards considéré comme un texte sacré, c'est-à-dire qui exigerait, comme l'écrivait Walter Benjamin dans «la Tâche du traducteur ", une traduction interlinéaire parce qu'en lui le sens et la lettre ne se laissent pas dissocier ${ }^{9}$, si le contact d'un tel texte, donc, n'entraîne pas chez le traducteur la remise en question de ce partage traditionnel des espaces et des pouvoirs entre lire et interpréter, d'une part, et traduire, d'autre part. Je voudrais donc esquisser ces quelques

8. Jacques Lacan, Écrits, Paris, Seuil, 1966, pp. 416-418. Cité dans les «Principes généraux », Traduire Freud, p. 21.

9. "Where a text is identical with truth or dogma, écrit Benjamin (et ce cas s'applique ici irrésistiblement au texte de Freud, du moins tel qu'il est perçu et transmis par l'équipe de Laplanche dans l'institution psychanalytique), where it is supposed to be "the true language" in all its literalness and without the mediation of meaning, this text is unconditionally translatable. [...] Just as, in the original, language and revelation are one without any tension, so the translation must be one with the original in the form of the interlinear version, in which literalness and freedom are united. For to some degree all great texts contain their potential translation between the lines; this is true to the highest degree of sacred writings. The interlinear version of the Scriptures is the prototype or ideal of all translation " (Walter Benjamin, "The Task of the Translator ", Illuminations, Edited and with an Introduction by Hannah Arendt, Fontana/Collins, 1982 [1973], p. 82). 
réflexions, plus théoriques que pratiques, sur la traduction en psychanalyse à partir des fantasmes qui se sont articulés autour de cette question dans trois ouvrages récemment parus qui illustrent de façon éloquente la diversité des positions transférentielles suscitées par le texte freudien : il s'agit d'abord, en tout droit tout honneur, de la position très tranchée adoptée par l'équipe de Laplanche à l'endroit de la note, dans son protocole éditorial, Traduire Freud; ensuite, de l'essai du traducteur allemand Georges-Arthur Goldschmidt, plus proche comme on le sait des vues exprimées par le groupe des traducteurs du Coq-Héron qui préconisent depuis longtemps, entre autres, la nécessité d'une édition bilingue des textes de Freud : cet essai s'intitule Quand Freud voit la mer. Freud et la langue allemande ${ }^{10}$, et Goldschmidt y développe une analyse comparée du français et de l'allemand, sur le plan de leurs ressources linguistiques, quant à la possibilité de traduire les concepts clés de la psychanalyse d'une langue à l'autre ; enfin, je m'appuierai sur l'ouvrage du psychanalyste Patrick Mahony, qui est l'un des premiers à avoir abordé dans ses textes en des termes analytiques cette question de la traduction dans le discours psychanalytique, et qui est également engagé depuis plusieurs années dans sa pratique discursive des textes de Freud dans la tâche de retraduction de l'édition anglaise de James Strachey, dont on se rend de plus en plus compte à quel point elle constitue précisément une interprétation du texte freudien : le dernier livre de Mahony s'intitule On Defining Freud's Discourse "I, et il y propose une nouvelle classification discursive de l'œuvre de Freud à partir de cette question du transfert à l'œuvre chez son lecteur ; cette typologie, même esquissée à grands traits, pourrait bien nous être également utile pour reprendre la question de la traduction.

\section{De la traduction comme art du tapissier}

Commençons donc par dire un mot du protocole de l'équipe de Laplanche dans lequel, incidemment, on sera frappé par le fait qu'il n'y ait, ni dans le glossaire proprement dit ni dans la «Liste des principaux termes n'ayant pas leur entrée propre» qui le complète, aucune entrée prévue pour le concept de traduction lui-même, ce qui ne laisse pas d'étonner puisque «conversion ", «translation/translater », "transposition" et "transformation " s'y trouvent, et qu'on a déjà souligné à maintes reprises à quel point ce concept de traduction,

10. Buchet/Chastel, 1988, 225 p.

11. Yale University Press, 1989,104 p. Je reprends plus loin, sous une forme différente, certains passages d'un compte rendu concernant ces deux ouvrages de Goldschmidt et de Mahony paru dans Spirale ("Cette langue venue du fond ", Spirale, $\mathrm{n}^{\circ} 90$, septembre 1989 , p. 8). 
Übersetzung, était central dans les écrits de Freud et inséparable, à cause de son étymologie et de son réseau sémantique même, des notions tout aussi capitales pour la théorie psychanalytique de métaphore et de transfert (Übertragung ${ }^{12}$ ). Cette omission de la problématique de la traduction dans un tel ouvrage programmatique qui cherche par ailleurs à prévoir toutes les objections qui pourraient lui être faites et qui entend surtout justifier envers et contre tous sa propre conception de la traduction, laisse le lecteur pour le moins rêveur. Or, il faut se demander s'il ne se passe pas également quelque chose de cet ordre relativement à la question de la note, dont l'usage est décrié à plusieurs reprises avec force par l'équipe de Laplanche tout au long de l'exposé de leurs règles et normes. On remarquera en effet que c'est à l'occasion d'un fort curieux passage concernant la retraduction des nombreuses citations incorporées dans le texte de Freud (en latin, en français, en anglais, en italien, etc.) que cette question de la note se trouve soudain problématisée. Dans ce passage, la traduction de l'original pose en effet plus clairement qu'ailleurs un problème «technique » en soulevant la situation en elle-même fort intéressante de l'intervention d'une tierce-langue, c'est-à-dire d'une "épreuve de l'étranger ${ }^{13}$ " encore plus visible dans ce cas, puisque la traduction ne pourra éviter de la rendre doublement étrangère, à elle-même d'abord, aux deux langues principales en jeu ensuite, en étant obligée de souligner la rupture produite par son interférence, et de mettre au jour le leurre d'un échange et d'une communication directes ayant lieu entre deux langues seulement, entre une langue d'origine (ou de départ) et une langue seconde (ou d'arrivée). Le lecteur se trouverait ainsi devant le seul cas qui autoriserait l'équipe des traducteurs, de leur propre aveu, à «donner en note les textes originaux indispensables». Suit alors cette vigoureuse mise au point quant à leur pratique de la note :

«En note », ... ? Serait-ce à dire que l'exactitude et la fidélité ne pourraient se passer de la glose, du justificatif et du commentaire? Affirmons nettement le contraire : le lecteur ne trouvera pas, consignée dans d'innombrables notes, la trace narcissique de nos états d'âme. Notre choix étant fait, nous faisons grâce au lecteur du débat intérieur et nous lui faisons confiance pour nous suivre, dans le cadre d'une cohérence de l'ensemble, qui fournit du texte, un véritable «tenant lieu ${ }^{14}$.

12. Voir Patrick Mahony, "Towards the Understanding of Translation in Psychoanalysis ", Meta, "Psychanalyse et traduction », $27: 1$, mars 1982, p. 64

13 J'emprunte cette expression à l'ouvrage séminal d'Antoine Berman sur la théorie de la traduction, l'Épreuve de l'étranger. Culture et traduction dans l'Allemagne romantique, Paris, Gallimard, "Essais ", 1984.

14. André Bourguignon, Pierre Cotet et al., op. cit., p. 56. 
Le ton péremptoire de l'affirmation, le mépris pour "la trace narcissique de nos états d'âme ", la parfaite maîtrise revendiquée ici hors de tout doute raisonnable seraient déjà en eux-mêmes assez alarmants si ne s'énonçait ici en outre, dans le droit fil d'une conception fondamentaliste et absolutiste de la traduction - c'est-à-dire absolument certaine de pouvoir rendre avec exactitude et fidélité la pensée de Freud du seul fait qu'elle écarte d'emblée tout contexte local et qu'elle postule au contraire un "cadre d'une cohérence de l'ensemble " (la traduction comme une sorte de bloc totalisant, monolithique, s'érigeant contre les " innombrables notes » qui pourraient la fragmenter, la disséminer) -, un modèle de l'œuvre comme totalité qui n'est jamais interrogé pour lui-même et encore moins remis en question et qui soutient de part en part ce programme de traduction ${ }^{15}$.

Ce commentaire sur la note ne manque pas de résonner à nouveau avec un écho tout particulier lorsque, un peu plus loin dans le texte, à propos de "l'art du traducteur" cette fois, on trouve une seconde affirmation de cette traduction pure, sans note ni glose, sans reste ni perte, qui est à l'origine de l'une des très rares comparaisons du texte et le fait sortir de son registre purement descriptif coutumier :

Pour comprendre une comparaison, nous sommes dans la situation d'un tapissier qui doit recouvrir, avec des rouleaux de papier peint d'une certaine largeur, un panneau de cloison dont la dimension n'est pas un multiple de celle des lés. De plus le papier peint est à motifs, exigeant aussi des " raccords » dans le sens vertical. Il serait simplement impossible, pour l'artisan, de faire fabriquer un papier aux dimensions du panneau - d'autant qu'il ne s'accorderait pas aux mesures des autres panneaux de la pièce. Tout l'art du tapissier est de faire passer les coupures et les raccordements dans les emplacements les moins visibles.

De même pour le traducteur freudologue : il ne forge pas un français germanique, mais s'entend à pratiquer les ruptures du signifiant et du signifié là où elles sont le moins gênantes, dans le cas précis de l'ouvre freudienne. [...] Cet art du traducteur, art du compromis assurément, ne saurait être assimilé à l'acceptation d'une défaite : nous affirmons que, dans chaque cas, comme pour le tapissier, il existe une meilleure solution possible ${ }^{16}$.

La comparaison mériterait d'être commentée jusque dans le détail du motif choisi, puisqu'on aura peut-être reconnu dans ce passage la

15. Il est en effet des plus douteux, lorsqu'on suit de près le parcours des "mots à l'œuvre " dans l'ensemble de l'œuvre freudienne, et même dans un seul texte, que ceux-ci se plient à cette règle d'une " cohérence de l'ensemble ", présupposé herméneutique qui est censé donner toute son autorité à ce glossaire. Comme le note Jacques Le Rider, avec de nombreux autres commentateurs, ce serait plutôt le contraire qui se produit, le contexte local et ponctuel l'emportant toujours dans le texte freudien sur la stratégie d'ensemble : "On ne peut pas non plus suivre cette école de traduction qui parle de "continuum " terminologique : il semble au contraire évident que les mêmes mots ont, suivant le contexte, un sens technique ou quotidien. " (art. cité. C'est moi qui souligne.)

16. Ibid., p. 56. 
retraduction d'une autre métaphore célèbre de Walter Benjamin par laquelle celui-ci évoquait la visée de la traduction à partir de l'image des débris d'une amphore, et faisait précisément appel à une tout autre conception de l'œuvre et de l'original, aux antipodes de celle qui est défendue par l'équipe de Laplanche :

Fragments of a vessel which are to be glued together must match, écrivait Benjamin, one another in the smallest details, although they need not be like one another. In the same way a translation, instead of resembling the meaning of the original, must lovingly and in detail incorporate the original's mode of signification, thus making both the original and the translation recognizable as fragments of a greater language, just as fragments are part of a vessel ${ }^{17}$.

On entrevoit ce qui joue, à travers la juxtaposition de ces deux passages concernant l'un l'art du traducteur, l'autre sa «tâche", d'une certaine image de la traduction, au sens fort du terme. Car ce qui différencie la traduction comme tapisserie chez Laplanche de la traduction comme fragment chez Benjamin, c'est d'abord l'affect qui affleure dans ces deux figures, triomphalisme et maîtrise sûre de savoir où couper, de pouvoir surmonter tous les obstacles et de parvenir à une «meilleure solution possible» dans tous les cas pour l'un, incorporation et infini travail du deuil, «dans un mouvement d'amour et jusque dans le détail " qui n'exclut pas la perte de l'objet et le désarroi devant la non-identité des parties pour l'autre. D'un côté, on affirme pouvoir restaurer le sens de l'original, pouvoir le « recouvrir» exactement en pratiquant les « ruptures du signifiant et du signifié là où elles sont les moins gênantes"; de l'autre, on renonce au principe d'identité des morceaux à reconstituer, on cesse de vouloir «se rendre semblable au sens de l'original» pour «incorporer » et donc faire passer dans sa propre langue non le sens du texte mais son mode de signification, son mode discursif ${ }^{18}$, c'est-à-dire sa manière de dire, au sens poétique

17. Walter Benjamin, op. cit., p. 78. Benjamin ajoute ensuite ceci, qui laisse à penser quant à la possibilité même de traduire le texte freudien tel que le conçoit l'équipe de Laplanche : "For this very reason translation must in large measure refrain from wanting to communicate something, from rendering the sense, and in this the original is important to it only insofar as it has already relieved the translator and his translation of the effort of assembling and expressing what is to be conveyed. " (lbid.)

18. La traduction comme translittération : comme le dit François Peraldi à propos de la traduction dans le discours psychanalytique, il s'agit de tenir compte "de la structure de signifiant du Vorstellung-Repräsentanz freudien » et de mettre « le signifié, le sens, entre parenthèses afin de mieux repérer et de dégager la logique qui prévaut $[\ldots]$. C'est donc plutôt une translittération que la compréhension freudienne semble impliquer et qu'il s'agirait effectivement d'ajouter, de substituer?, à une conception de la traduction toujours encombrée du sens : une translittération qui ouvrirait, en quelque sorte, le champ d'une interprétation véritablement psychanalytique [...]". ("Présentation ", Meta, $27: 1$, mars 1982, pp. 60-61). 
de la Dichtung allemande. (Remarquons au passage que Freud traducteur de Charcot était lui-même très sensible à cette qualité particulière de la parole lorsqu'il déclarait dans sa "Préface » à la traduction des Leçons du mardi : «Dans la traduction de ces leçons, je me suis efforcé - non pas d'imiter le style incomparablement clair et en même temps si noble de Charcot, ce qui m'aurait été impossible -, mais d'effacer le moins possible leur allure de libre parole $\left.{ }^{19} . »\right)$

Ainsi, tout en semblant dire la même chose, ces deux images disent-elles tout autre chose sur la "tâche" du traducteur, très vite convertie (sublimée ?) en "art " par l'équipe de Laplanche. La traduction-tapisserie de Laplanche cherche à effacer le plus possible les démarcations et à faire "coller" les morceaux en fonction du sens, alors que la traduction selon Benjamin consiste au contraire à renoncer à la coïncidence des coutures ou à seulement transmettre le sens c'est justement là à ses yeux l'inessentiel de la traduction, ce qui la rend inévitablement inférieure. Il s'agit pour lui, tout au contraire de l'équipe de Laplanche, non de disjoindre le signifié du signifiant, mais bien de les envelopper dans la traduction, pour filer une autre métaphore célèbre: "While content and language form a certain unity in the original, like a fruit and its skin, the language of the translation envelops its content like a royal robe with ample folds ${ }^{20}$. "Sans qu'il soit possible de « déplier » davantage ici cette métaphore de l'enveloppement, disons seulement en ce qui concerne la traduction du texte de Freud qui nous retient, qu'il ne nous semble pas que cette traduction, du moins dans la version de l'équipe de Laplanche, parvienne à atteindre la souveraineté, l'expansion, la liberté dont parle Benjamin, à cause notamment du transfert particulier contracté à l'endroit du texte de Freud compris comme stricte reproduction de l'intentio de l'original, comme tâche de fidélité par rapport au sens à transmettre, et non d'émancipation ou de croissance par rapport à celui-ci.

\section{Terminologie "raisonnée" et angoisse du traducteur}

Sans chercher à ajuster davantage les pièces de mon propre commentaire-mosaïque, je retourne donc pour l'instant une dernière fois au protocole éditorial de Laplanche pour suivre jusqu'au bout le fil concernant cet «art du traducteur » qui me retient plus particulièrement dans ce contexte où se trouve explicitement discutée la portée de la note. Si l'on suit l'appel de note, on lit cette note en bas de page: "Sur l'opposition entre "la tâche du traducteur» (selon l'expression de W. Benjamin) et la liberté du commentateur, voir plus loin le

19. Sigmund Freud, "Préface à la traduction des «Leçons du mardi " de Charcot " [juin 1892]. l'Écrit du temps, no 7, été 1984, p. 58.

20. Walter Benjamin, op. cit., p. 75 . 
«post-scriptum » à l'article « angoisse » de la terminologie raisonnée ${ }^{21}$ », qui renvoie le lecteur à l'article «Angoisse» dans le glossaire où sera justifiée de façon paradoxale cette condamnation de la note. Et cela se passe de commentaire, je crois, que ce soit précisément cet article-là qui laisse revenir l'angoisse qui était refoulée par le ton jusque-là triomphant du traducteur affirmant pouvoir se passer « de la glose, du justificatif et du commentaire ", et qui laisse soudain affleurer, dans une situation textuelle qui sort littéralement du "cadre de cohérence de l'ensemble " posé, une certaine défaite du traducteur et même une certaine irrationalité, une certaine incohérence au sein même des principes et des justifications.

L'article "Angoisse» est en effet le lieu d'une mise au point quant au statut de la note dans cette édition, qui sera réduite au minimum et ne "[comprendra], volontairement, aucun commentaire de fond, rapprochement des textes, historique des concepts, etc. ${ }^{22}$ ". Or, justement, cette note en "post-scriptum" de l'article "Angoisse" constitue une triple infraction à la règle, en faisant tout ce que la note n'est pas supposée faire ici (un commentaire de fond sur la distinction entre «angoisse " (Angst) et "peur » (Furcht), un rapprochement avec le texte de Laplanche, portant sur l'Angoisse ${ }^{23}$, et un historique du concept). En outre, et c'est sur ce point que j'aimerais insister, ce supplément, ce "p.-s. » qui est bien une véritable «N.d.T. », est aussi l'occasion d'une nouvelle profession de foi dans la tâche du traducteur, dont il est dit que «face à la terminologie, [elle] ne saurait participer de la liberté d'expression du commentateur $[\ldots]^{24}$ ». De façon révélatrice, la profession de foi en faveur de la traduction, contre le commentaire et l'interprétation, est l'occasion d'une nouvelle manifestation affective et polémique quant au rôle du traducteur freudien qui, dans une véritable explosion de colère, perd ici toute "neutralité" et passe à l'attaque :

Depuis plus de vingt ans que, dans nos ouvrages, nous discutons et mettons "au travail" les textes de Freud, nous ne nous sentons pas moins à même que tant d'autres de commenter pertinemment les résonances et les ambiguittés de la langue freudienne et de nous extasier devant son caractère " fragile " et « intraduisible ». Nous regrettons seulement que tant de commentaires portant sur les " mots » de Freud ou sur sa « langue » soient, le plus souvent, peu avertis d'un troisième élément non moins essentiel : sa pensée ${ }^{25}$.

21. André Bourguignon, Pierre Cotet et al., op. cit., p. 56.

22. Ibid., p. 66.

23. Jean Laplanche, Problématiques I. L'angoisse, Paris, Presses universitaires de France, 1980.

24. André Bourguignon, Pierre Cotet et al., op. cit., p. 80 .

25. Ibid., p. 81 . 
Voilà donc que cette fois, et cette fois seulement, car la brèche ouverte par cette note dans un système de traduction par ailleurs imparable ne se répétera pas, le traducteur doit battre en retraite et céder le pas au commentateur: "L'auteur de ces lignes ne peut mieux faire ici que renvoyer à son ouvrage sur "L'angoisse " et notamment aux pages 49 à $71^{26}$.» Ce renvoi est fort instructif, il est tout sauf anecdotique à mon avis, parce que, ce faisant, le traducteur freudologue se replie défensivement sur sa fidélité à la «pensée » de Freud, ce qui laisse entendre que ceux qui s'attachent aux " mots" ou à la "langue » de Freud ne savent littéralement pas de quoi ils parlent, et surtout que le style et la conceptualité freudienne sont restés, malgré qu'il en ait, dissociés à ses yeux (tout comme d'ailleurs, dans le passage déjà cité plus haut, le signifiant et le signifié). Ce «p.-s. » fonctionne donc selon le mode de la dénégation et remet finalement en question tout le système sur lequel il se greffe. C'est une chose en effet de vouloir limiter à tout prix la portée de la note dans la traduction, mais c'est un événement non négligeable que d'avoir à s'en expliquer dans un ouvrage qui, lui-même publié hors série et voué à disparaître ${ }^{27}$, est tout entier de l'ordre du supplément. Il y va ici d'une véritable situation aporétique, car qu'est-ce en effet que ce glossaire, instrument indispensable pour comprendre les principes de traduction adoptés pour ces CEuvres complètes de Freud. Psychanalyse, sinon une pratique extensive de la note qui fait la preuve que cette traduction ne peut en aucun cas s'autosuffire sans justificatif? Plus qu'aucune autre peut-être, la traduction de l'équipe de Laplanche ne peut en effet se passer de la glose qu'elle déclare pourtant rejeter. Paradoxalement, le glossaire, loin d'établir une fois pour toutes les principaux concepts freudiens, oblige plutôt le lecteur à constamment retraduire le texte qu'il a sous les yeux.

Par ailleurs, ce renvoi à «l'auteur de ces lignes» (Laplanche, en l'occurrence) est encore important pour une autre raison, notamment parce qu'il laisse également revenir une autre question, complètement occultée par ce nouveau concept de traduction en équipe, c'est-à-dire la notion même d'auteur, mieux, de sujet-traducteur. Or, il faut se demander si l'on n'aborde pas là l'aspect fondamental qui rend cette traduction collectiviste si peu convaincante, si peu "parlante » pour le lecteur: la question du transfert, constamment bloqué ou neutralisé par tout refus de la subjectivité et, partant, de la signature d'un véritable sujet-traducteur, au sens fort du terme, s'engageant en son nom et en son style propres envers le texte.

\section{Ibid., p. 80.}

27. On lit en effet dans l'" Avertissement de l'éditeur " que "Cet ouvrage n'est pas voué à la pérennité, car son contenu sera ultérieurement réparti entre le tome I et le tome XXI des OCF. $P$ ». 
(Mais de quel nom propre se sert le traducteur? Des nuances capitales s'imposeraient à ce sujet, que je ne peux développer ici faute d'espace. Disons seulement que le contrat qui lie le traducteur au texte joue d'abord dans le registre de l'imaginaire, ce qui revient à accepter sa tâche comme fiction, effet de vérité, et non à se faire le serviteur de la vérité du texte, dans l'ordre du réel : mais cela, c'est une autre histoire du transfert du traducteur, qui reste à élaborer.)

Car, à découper l'acte de traduction en autant d'opérations à la chaîne, à l'aligner sur le modèle universellement régnant de la cybernétique - le feed-back semble en effet le mot clé de cette entreprise où Janine Altounian elle-même est présentée comme la "mémoire" du groupe 28 -, on (un « on » impersonnel et sans narcissisme) cherche à effacer dans l'anonymat d'une «vision unitaire» le style et les marques transférentielles de chaque traducteur individuel :

Les contributions de chacun sont devenues indiscernables, le multiple est devenu un et les parties se sont agrégées en un tout cohérent. Cette lente maturation d'un corps homogène, alors que l'hétérogénéité initiale faisait craindre qu'il ne vit jamais le jour, se poursuit dans un respect mutuel et par auto-organisation progressive, réalisant l'unité d'un organisme complexe et performant ${ }^{29}$.

\section{De la traduction comme dérivé : explorations génétiques de l'inconscient à l'œuvre entre les langues}

À l'opposé de cette conception dogmatique, homogénéisante et pour tout dire quasi théologique de la traduction (le «multiple est devenu un»), où la note est de toute nécessité sacrifiée comme lieu d'épanchement de la subjectivité du traducteur et l'accent porté sur une performance plus mécanique (machinique même) que vraiment organique, on a vu récemment se déployer un tout autre fantasme de traduction, dont le récent livre de Georges-Arthur Goldschmidt, Quand Freud voit la mer, traduit au mieux le symptôme. Goldschmidt rassemble dans cet essai des notes et réflexions ponctuelles sur la traduction de certains concepts clés de la psychanalyse (on lira, par exemple, avec profit ses importantes remarques sur das Unbewusste, dont l'« inconscient » rend si mal en français le caractère «insu » et le «genre étrange et pénétrant du neutre ${ }^{30}$ » allemand; sur die Verdrängung / le « refoule-

28. Ibid., p. 69. On sait que, sous la direction de Jean Laplanche, Pierre Cotet et André Bourguignon, l'équipe s'est partagée la " tâche du traducteur" selon une stricte division, quasi tayloriste, du travail : chaque spécialiste (freudologue, germaniste, terminologue, réviseur, etc.) avait sa part dans la chaîne, et tout différend était soumis au consensus du groupe ou, à défaut, à la décision finale de Laplanche.

29. Ibid. p. 70.

30. Georges-Arthur Goldschmidt, op. cit., p. 35. 
ment », qui porte cette particule capitale («ver») du lexique freudien ; sur le chassé-croisé entre Trieb et "désir", termes qui n'ont pas, à strictement parler, d'équivalents d'une langue à l'autre; ou encore sa discussion de cette autre notion «intraduisible» en français : See / «âme»). Goldschmidt poursuit également dans ce livre une interrogation de portée plus générale et ambitieuse sur l'inconscient à l'œuvre entre les langues. Ces commentaires sur la langue allemande sont présentés ici juxtaposés de façon parataxique plutôt que vraiment articulés, et ils ne sont pas sans rappeler, toutes proportions gardées, une certaine tradition allemande de la glose philologique, à cause notamment du recours constant qui est fait par Goldschmidt aux dictionnaires étymologiques comme à une réserve originaire porteuse de la vérité de la langue.

Mais il ne s'agit pas ici que d'érudition, car ce livre s'inscrit aussi à point nommé dans la polémique actuelle autour de la traduction de Freud. Il n'est par exemple que de lire la "Note» placée en ouverture de ce livre pour s'en convaincre et mesurer l'écart qui sépare irrémédiablement la position de Goldschmidt de celle qui est mise en œuvre par l'équipe de Laplanche dans son protocole. "Tous les termes allemands employés dans cet ouvrage, déclare Goldschmidt, sont traduits au fur et à mesure de leur emploi. Tous les passages cités de Freud ou d'autres auteurs de langue allemande sont retraduits par G.-A. G. ". Ces deux petites phrases laissent clairement entendre ce qui constituera l'enjeu théorique déployé dans la suite du livre, soit, d'une part, la mise en cause radicale de toute traduction effectuée dans un "cadre d'ensemble" où la portée contextuelle des connotations suscitées par un mot, chutant en un point précis du texte freudien, serait négligée au profit de la seule logique du sens. D'autre part, Goldschmidt s'élève ici laconiquement contre toute idée d'un glossaire de termes pré-traduits qui se substituerait au geste d'un sujet-traducteur particulier; il remet ainsi en cause l'usage courant et la loi implicite qui obligent le traducteur à plier devant telle traduction déjà établie faisant autorité. Bref, Goldschmidt affirme avec force le droit, sinon la nécessité, pour tout traducteur qui veut vraiment faire le saut qu'exige la traduction (Goldschmidt rappelle qu'étymologiquement Übersetzung veut aussi dire : sauter par-dessus un abîme) à retraduire les passages cités. Loin de lire cette mise au point comme une revendication innocente et subjectiviste, on sera frappé par la volonté de porter au jour des aspects de la tâche du traducteur qui restent, à tort, voilés sous le masque bienséant ou ancillaire du retrait et de l'effacement, comme s'ils étaient toujours trop impudiques pour être abordés de front.

Contre la conception de la traduction défendue par l'équipe de Laplanche comme transmission d'un sens intact et transparent, Goldschmidt propose ici une autre façon de voir les rapports de Freud à 
la langue allemande et, partant, à la traduction elle-même où «Il faudrait, dit-il, pour chaque mot, indiquer les variations possibles, les rapports ou l'absence de rapports avec le français ${ }^{31}$. " On voit tout de suite à quel point une remarque de ce genre, qui substitue au procès de la traduction comme décision ${ }^{32}$ une autre pratique formelle et syntaxique où l'indécidable garderait sa chance, s'inscrit en dehors de toute préoccupation d'ordre pragmatique, en mettant directement en cause le principe d'économie de la traduction déjà évoqué, et s'éloigne d'une traduction « raisonnée » comme celle qui est pratiquée par l'équipe de Laplanche, vers une traduction intralinguale qui retrouve sous les mots et le lexique proprement dit le flux de la langue. Il faut cependant remarquer que certains dangers guettent aussi cette conception de la traduction, bien proche de fait de la paraphrase et courant souvent le risque d'une infinie régression, comme le suggère déjà la métaphore marine qui la soutient. Sa force réside cependant dans la modification radicale qu'elle fait subir au contrat qui lie le traducteur au texte freudien, contrat qui n'est plus alors de stricte restitution scientifique, de resémantisation et de reconstruction de la conceptualité freudienne comme ce que l'on trouve dans le modèle Laplanche, mais qui relève plutôt d'une exploration anasémique ${ }^{33}$ remontant en aval de la traduction proprement dite. Le traducteur du texte freudien est alors à l'écoute de l'inconscient linguistique à «fleur de mots" et des différences entre langues qui disent la même chose, mais selon des voies toutes différentes.

D'avance pris en son piège, la langue (il n'est langage que pour cela) " fait voir" ce dont il (sic) parle mais il le fait voir au travers de lui. Ce que l'on voit n'est, de plus, qu'une coloration de la mer. Le texte

31. Ibid., p. 30.

32. Benjamin soulève aussi cette question lorsqu'il se demande, après avoir évoqué le problème, à ses yeux insoluble, de la maturation, de la croissance du "noyau de pur langage" que doit viser la traduction, si le sol ne se dérobe pas sous les pieds du traducteur dès lors qu'il abandonne la reproduction du sens comme son unique but: "For is not the ground cut from under such a solution if the reproduction of the sense ceases to be decisive?" (Op. cit., p. 77.)

33. À la suite de Nicolas Abraham dans l'Écorce et le noyau, Jacques Derrida définit l'anasémie comme "la remontée en deçà du sens vers des concepts qui sont non seulement originaires mais pré-originaires. [...] Quand [...] Freud parle de ceci ou de cela, des grands concepts psychanalytiques, il ne les entend pas dans le sens courant de la langue [...] il faut remonter (d'où le sens, d'où le mot de " anasémie ") en deçà du sens pour comprendre comment le sens s'est formé " ("Tables rondes sur la traduction", l'Oreille de l'autre. Otobiographies, transferts, traductions. Textes et débats avec Jacques Derrida, sous la direction de Claude Lévesque et Christie V. McDonald, Montréal, VLB éditeur, 1982, pp. 144-145). Commentant lui aussi le choix de Freud d'employer des mots usuels pour leur faire dire autre chose, François Peraldi dira que la spécificité de la traduction psychanalytique "fonctionne comme une sorte de traduction intralinguale dont la fonction serait d'évidement du sens ». (Ibid., p. 173. Je souligne.) 
de Freud ainsi « dérive" de ce dont il parle et Freud, qui le sait mieux que personne, ne cesse de redire que jamais rien ne vient à la surface tel que cela fut dans les profondeurs (tout le problème de la psychanalyse est peut-être là). Les textes de Freud en somme ne cessent de parler de ce dont ils proviennent mais cela ils ne le sont pas. Tout texte "dérive » ce dont il parle, il le déplace. Tout texte est traduction, il est donc inadéquation de base. Pourquoi voudrait-on que la traduction coïncidât avec le texte puisque le signe déjà ne coïncide pas avec ce qu'il représente (sans quoi il y aurait la chose et non le signe) ? La situation de Freud par rapport à son dire est semblable à celle du traducteur par rapport à Freud ${ }^{34}$.

$\mathrm{Si}$ tout texte est déjà, dans une certaine mesure, traduction, "dérive " dont le point d'origine est à jamais perdu, "inadéquation de base " par rapport à tout fantasme de coïncidence et d'identité avec l'original, on commence à entrevoir qu'une traduction au mot à mot ${ }^{35}$ ne saurait suffire à la tâche dans le cas du texte freudien et qu'il faut, au contraire, pour pouvoir retrouver la cohérence de sa démarche, que le traducteur ne se contente pas d'une seule traduction interlinguale, au sens courant du terme, mais encore suive les détours du français, fasse le tour des obstacles et des insights - et j'irai jusqu'à dire des Einfällen, des pensées incidentes - qui lui sont particuliers et qui peuvent à tout moment lui venir, lui tomber dessus. "Et si le «sens ", demande Goldschmidt, était d'être dans deux langues et si toute la démarche de la psychanalyse consistait à faire voir l'autre dans la langue? Qu'une langue est en somme l'analyse de l'autre? L'allemand (et réciproquement) analyse, porte au jour le caché du français [...]. Une langue est le recours de l'autre, elle est son halètement devant l'impossible expression par elle-même ${ }^{36}$ ».

Toutefois, bien qu'elle soit éminemment séduisante, il faut se demander si cette hypothèse d'un inconscient linguistique à l'œuvre entre les langues, où une langue supplée l'autre - «comme si le français disait ce que ne dit pas l'allemand et l'inverse, car ce que l'allemand met au-dedans (erinnern) c'est ce que le français rappelle, comme si une langue commençait là où l'autre se termine ${ }^{37}$ »-, si ce jeu de "tire-pousse", pour reprendre l'expression de Goldschmidt ( « En somme, une langue - le français - , tire ce qu'une autre -

34. Georges-Arthur Goldschmidt, op. cit., p. 49.

35. Darius Ornston fait une remarque qui va dans le même sens à propos de la Standard Edition de James Strachey : voir " Freud's Conception is different from Strachey's ", The Journal of American Psychoanalytical Association, 33, 1985, pp. 337-370, et « Strachey's Influence », International Journal of Psycho-Analysis, 63, 1982, pp. 409-426.

36. Georges-Arthur Goldschmidt, op. cit., pp. 59-60.

37. Ibid., p. 90. 
l'allemand -, pousse ${ }^{38}$ ), sont suffisants pour faire disparaître l'affect d'angoisse, le malaise du traducteur freudien devant sa tâche et faire de celle-ci un travail « des plus utiles et des plus "gratifiants» 39 ». Ce fantasme d'un chevauchement des langues, d'un emboîtement inconscient se produisant entre les langues précisément là où elles se ratent et manquent à dire est peut-être surtout une manière d'esquiver le désarroi (travail du deuil conviendrait mieux) du traducteur. Ce qui se fait clair par contre dans l'essai de Goldschmidt, de glissements en dérives, en inévitables retours et répétitions ${ }^{40}$, c'est que cette métaphore marine apparaît surtout comme l'expression d'un autre fantasme transférentiel du traducteur freudien, qui prend ici la forme d'un désir de fusion et de régression par rapport à la question du sens et des contenus proprement dits de la vie psychique, qui échapperont toujours d'ailleurs aux limites imposées par le lexique d'une langue, quelle qu'elle soit. Dans une telle logique de la traduction comme réécriture du texte freudien ${ }^{41}$, la note du traducteur cesse par ailleurs d'être utilisée dans un registre restreint pour, au contraire, faire l'objet d'un usage généralisé, et la traduction est alors elle-même inséparable du commentaire ou de la glose explicative.

On pourrait bien entendu amorcer une critique du fantasme originaire, du fantasme des origines, qui joue un rôle théorique non négligeable, mais non complètement analysé à mon avis, à l'arrière-plan de cette conception de la traduction, sous la forme du leurre étymologique tout particulièrement : "On peut se demander si toute l'étymologie n'a pas précisément pour but d'élucider les feintes du langage, d'y (sic) parer afin d'éviter d'être surpris par lui ; remonter à l'étymologie, c'est tenter d'éviter que le langage ne parle à la place de qui l'em-

38. Ibid., p. 90.

39. Ibid., p. 151 .

40. À la fois description cognitive et image poétique de la langue allemande tout au long de cet essai, la métaphore marine est en effet si omniprésente qu'elle conduit peu à peu le lecteur à se demander où passe la frontière entre l'exploration et l'exploitation d'une figure rhétorique. S'il est acquis, comme l'a montré à maintes reprises Judith Schlanger dans ses ouvrages sur l'invention intellectuelle, que la métaphore est d'ores et déjà porteuse de savoir, peut-on tout de même abuser d'une figure, même si celle-ci prend, comme la mer, la forme de ce qui n'a précisément pas de fond?

41. "Si Freud avait écrit en français, il est évident qu'il s'y serait pris tout autrement pour arriver à la même chose, son parcours à travers la mer aurait été autre. C'est pourquoi il n'est peut-être pas sûr que les traductions puissent totalement rendre compte de ce qu'il voulait dire et peut-être vaut-il mieux " récrire * Freud que de le traduire, ce qui naturellement est impossible " (G.-A. Goldschmidt, op. cit., p. 170). On remarquera que la fin de la phrase réintroduit une ambiguitté : est-ce la traduction ou la réécriture qui s'avère " naturellement $[. .$.$] impossible " ?$ 
ploie $^{42}$.» On croit ainsi pouvoir «retourner au sens premier des mots», «ramener la langue à son sens premier ${ }^{43}$ » en faisant usage des dictionnaires de la langue allemande (le Wahrig, le Kluge : Etymologisches Wörterbuch der deutschen Sprache) : la naïveté (sans acception péjorative) - et la nativité - de cette position dit assez clairement, je crois, à quel point elle est une construction fantasmatique du sujet traducteur, une rêverie vocalique qui revient le hanter, le tenter. Car il ne suffit évidemment pas de suggérer, comme le fait Goldschmidt, que «Tout le travail de Freud pourrait, en somme, lui avoir été soufflé par sa langue maternelle et par les courants qui, dans cette mer, vont de rive en rive. Dans une langue, comme dans la mer, tout passe de l'un à l'autre $[\ldots]^{44}$ », il ne suffit pas de rappeler que l'allemand n'est pas, comme on l'entend dire un peu trop souvent par les temps qui courent, plus rugueux, plus lourd, plus martelé que le français, qu'il est au contraire une langue toute fluide et spatiale qui «se forme à partir de l'emplacement et du mouvement dans l'espace 45 ", une langue de part en part concrète (Goldschmidt attaque ici au passage le préjugé linguistique qui la fait résonner, du moins à certaines oreilles françaises, comme une langue hautement abstraite, intellectualisée, désincarnée, entendre philosophique), bref, une langue lié aux gestes et aux désirs du corps, une langue du fort-da, du flux et du reflux, où le sens érotique originel des mots ne cesse de remonter à la surface. Il ne suffit pas de remarquer que plusieurs des termes utilisés par Freud appartenaient au langage courant ou se retrouvaient déjà en d'innombrables passages de l'œuvre de Goethe par exemple pour conclure que « tout se passe comme si toute la littérature allemande [...] avait préparé la voie à Freud. Tout se passe comme s'il n'avait eu qu'à puiser dans les textes et sous ses yeux mêmes, dans la langue ${ }^{46} \%$. La découverte

42. Ibid., p. 140. L'essentiel de la critique que nous pourrions adresser à Goldschmidt recouperait la définition de la traduction psychanalytique comme anasémie (voir ci-dessus, note 33) : la remontée étymologique, originaire, dans les "flots" de la langue, n'entraine pas automatiquement une remontée anasémique, préoriginaire, d'évidement du sens. L'une et l'autre ne devraient donc pas être confondues.

43. Ibid., p. 184.

44. Ibid., p. 133.

45. Ibid., p. 17.

46. Ibid., p. 179. Il est pour le moins excessif de tout centrer sur la langue, comme le fait Goldschmidt à plusieurs reprises : "On le voit, Freud ne fait que répéter ce que dit la langue, faisant attention comme personne à ce qu'elle dit, il se contente de parler après elle, de la "faire parler", comme le dit si joliment le français » (ibid., p. 81) ; ou encore cet autre passage : "On ne le redira jamais assez à quel point Freud a regardé faire la langue. Tout se passe, à le lire, comme si, malgré la rigueur exemplaire de la construction de ses textes, il laissait se déployer la langue, comme s'il la laissait faire [...]» (ibid., p. 96). L'intuition de Goldschmidt est peut-être juste, mais sa formulation laisse surtout entendre sa propre fascination à l'endroit de la langue-mère, sa tentation de s'y perdre, de se laisser submerger par elle... 
freudienne ne tient pas pour l'essentiel au vocabulaire ou au lexique, mais plutôt à un style, à une syntaxe; elle est moins une question de langue que de langage, comme le disait Lacan (ces deux aspects sont ici constamment confondus). Autrement dit, il s'agit moins pour le sujet freudien de s'assujettir à la langue commune, même dans sa dimension "insue ", que d'y frayer, à travers toutes sortes de détours et de détournements de sens, sa voie propre. En se demandant si "l'inconscient n'est pas totalement tributaire du langage ${ }^{47}$ ", Goldschmidt va, nous semble-t-il, trop loin, et surtout fait la part trop belle à la langue, qui ne dit rien d'elle-même. S'il est irréfutable que "C'est dans l'allemand que Freud place son discours ${ }^{48}$ ", il est encore plus essentiel de rappeler que «la portée même de l'œuvre de Freud vient pourtant de ce qu'elle n'est pas particulière à une langue, l'allemand $[\ldots]^{49} "$ : on aurait souhaité que l'auteur s'explique mieux sur ce paradoxe qui fait au texte freudien une situation si inouïe dans l'histoire de la traduction, la rendant du même coup impossible et si nécessaire à traduire. Comme le disait Jacques Derrida à propos de tout texte sacré - texte qui est sacré dès qu'en lui se manifeste du propre, de l'intraduisible -, un texte «ne sur-vit que s'il est à la fois traductible et intraductible. [...] Totalement traductible, il disparaît comme texte, comme écriture, comme corps de langue. Totalement intraduisible, même à l'intérieur de ce que l'on croit être une langue, il meurt aussitôt ${ }^{50}$ ». Les récentes traductions de Freud n'ont peut-être pas assez pris la mesure, à la fois pratique et théorique, de cette double contrainte... Par ailleurs, l'analyse comparée du français et de l'allemand menée ici, même si elle est loin d'être toujours elle-même dénuée de l'esprit de clan qu'elle dénonce du côté français, en dépit de ses dangers et de ses limites, demeure une approche très riche pour saisir les transferts à l'œuvre dans la traduction ${ }^{51}$.

\section{De la traduction comme géologie : suivre les failles du texte freudien}

Je voudrais maintenant pour finir dire brièvement un mot de la nouvelle classification des écrits de Freud proposée par Patrick Mahony dans

\footnotetext{
47. lbid., p. 109.

48. Ibid., p. 69.

49. Ibid., p. 32.

50. Jacques Derrida, op. cit., p. 169.

51. Voir, par exemple, l'analyse risquée mais très suggestive que Goldschmidt élabore, il est vrai trop elliptiquement, à partir de l'adjectif «untertan " qui occupe une place incalculable dans l'inconscient linguistique allemand, et dont il retrace quelques points d'affleurement, de Hölderlin, au Président Schreber, à l'Homme aux rats, à Paul Celan, et jusqu'à l'insoutenable "perversion du langage " qu'a constituée la folie nazie.
} 
son dernier livre, On Defining Freud's Discourse, dans lequel il poursuit la relecture des récits de cas de Freud déjà amorcée dans Freud as a Writer et Freud and the Rat Man, en suivant le double fil de l'exposition discursive et de l'élaboration théorique toujours étroitement entrelacées dans le discours freudien. Mahony reprend cette analyse textuelle à propos de deux textes de Freud, composés à près de cinquante ans d'intervalle, et qui marquent ainsi les points extrêmes de son parcours, l'un frais et plein d'espoir, se situant à l'aube de la psychanalyse naissante (il s'agit du cas de Katharina, l'une des figures les moins connues des Études sur l'hystérie, dont Freud rédigea le récit clinique en 1893-1895: au cours d'une excursion en montagne pendant ses vacances, alors qu'il reprenait souffle dans un relais, Freud avait "guéri » cette jeune fille souffrant d'hystérie en une seule séance de quelques heures); l'autre texte baigne dans une lumière beaucoup plus sombre, crépusculaire si l'on peut dire, puisque c'est un Freud vieilli, malade et beaucoup plus pessimiste quant à l'avenir de la psychanalyse, qui signe ici l'un de ses derniers grands textes, «Analyse avec fin et sans fin 52 \%.

Recontextualisant avec sa minutie habituelle l'arrière-plan historique de ces textes, Mahony examine l'écriture de Freud dans ces deux cas si contrastés et propose une nouvelle classification des écrits de Freud, qui devraient être, selon lui, moins regroupés chronologiquement, par sujet ou par genre, que selon leur mode discursif : dogmatique ou génétique. Précisons que, pas plus que les pulsions de mort et de vie dans "Analyse avec fin et sans fin", ces deux modes ne s'opposent tout uniment, mais qu'ils s'associent plutôt et se mélangent, à des degrés variables, à l'intérieur d'un même texte. Ainsi, dans le récit de cas de Katharina, même si son mode d'exposition est dans l'ensemble plutôt formel, Freud est aussi amené à constamment érotiser son discours, notamment par l'usage essentiel des déictiques ("hereand-now", "there-and-then") qui révèlent tout à tour sa distance et sa proximité à l'égard de sa jeune analysée et laissent clairement entrevoir à quel point cette expérience analytique est tout aussi désorientante pour lui et menace de lui faire littéralement perdre pied. Or,

52. On sait que, dès le titre, ce texte de Freud soulève lui-même des problèmes « interminables » de traduction : je reprends pour ma part la traduction récemment proposée par Monique Schneider dans le Trauma et la filiation paradoxale (Paris, Ramsay, "Psychanalyse ", 1989), qui recoupe également la discussion de Patrick Mahony sur les connotations autour de "endlich": citant un manuscrit de Helmut Junker ("The connotation of endlich can be "ending", "a process of ending ", "having ended", "being limited, finite and final " - so how do we translate that term? [...] A convenient literal translation into English would be "the ending and the non-ending analysis", giving a flow, a mouvement of thought, interwoven and without a chance of logical explication and definition " (op. cit., p. 79). Mahony suggère de traduire le titre de l'essai de Freud par: "Analysis, its endingness and unendingness". 
pour entendre ce jeu subtil des déictiques (contre)transférentiels, la nécessité d'une traduction nuancée s'impose, et force est de constater que celle de Strachey, constamment neutralisante, masque totalement cet aspect capital du texte de Freud. La problématique de la traduction se retrouve donc également au cœur de cet ouvrage de Mahony, puisque lire Freud c'est d'abord le traduire, et le retraduire, car il s'agit de corriger les nombreuses distorsions et omissions de la version de Strachey qui a toujours tendance, comme le montre de façon convaincante Mahony, à limiter le côté fluctuant, impulsif et expérimental du texte de Freud, c'est-à-dire à atténuer tout ce qui dans son style même conserve vives les traces des processus primaires.

Cette question du transfert à l'œuvre dans la traduction même du texte freudien est peut-être illustrée de façon encore plus exemplaire dans la «construction» d'un texte comme «Analyse avec fin et sans fin ", dont l'organisation " interminable " reste elle-même ouverte, déliée, inachevée (Mahony parle de "loose design", d'une structure générale qui est «slack ${ }^{53}$ ). Le mode de discours génétique que Freud pratique dans ce texte l'emporte de loin sur tout souci d'exposition dogmatique et l'engage à privilégier des connexions "rayonnantes" (radial) plutôt que simplement latérales. "Freud talked about process in a processive way ${ }^{54}$ ", note Mahony. Par mimétisme avec la déliaison du texte freudien sans doute, cette partie de son essai est plus elliptique et fonctionne elle aussi, en sériant des exemples sans les lier entre eux, comme un mobile d'associations qui met en relief ce trait marquant $\mathrm{du}$ rapport de Freud à la langue, "the purpose of which, commente Mahony, is to bypass the limitations of an incomplete science and to escape from the confines of the lexicon, whose more or less fixed and univocal meanings are not adequate to the processive aspects of psychic life ${ }^{55}$ ".

Ce qui nous intéresse tout particulièrement ici, c'est de voir à quel point la liberté plus ou moins grande que Freud s'accordera dans la composition de ses textes, entrainera également une fluctuation dans le transfert que le lecteur, et à plus forte raison le traducteur, entretiendront à son endroit. Ainsi la structuration génétique d'« Analyse avec fin et sans fin» favorise-t-elle un certain jeu des associations chez le lecteur à cause de son indétermination même, alors que la structure plus dogmatique du récit de cas de Katharina suscite un détachement ironique beaucoup plus grand chez le lecteur à cause de la distance de Freud lui-même. La condition d'une bonne lecture de Freud - et la traduction est bien l'une des formes les plus raffinées, supérieures

53. Patrick Mahony, op. cit., p. 69.

54. lbid., p. 67.

55. Ibid., p. 85. 
de la lecture - réside donc dans une large mesure dans la capacité du lecteur-traducteur de saisir ces effets transférentiels. On appréciera d'autant plus - le fait est assez rare pour qu'on le souligne - que Mahony livre lui aussi quelque chose de sa propre expérience de lecture et de traduction du texte freudien :

If one presumes that Freud's texts are dogmatically self-sufficient and complete [c'est bien là le présupposé de l'équipe de Laplanche], one is starting with an enormous handicap and may never arrive at the point of integrating into one's reading the hermeneutics of suspicion that Freud, along with Nietzsche and Marx, contributed to modern culture. My own approach presumes that Freud's texts, like crystals, have fault lines, and I wonder where they are and how wittingly as well as unwittingly they are covered up. I muse about whether Freud's self-irony fully accounts for the gaps of contradictions in his report. Given the foiling nature of the unconscious, these questions are where I begin, not end, my inquiry. In addition, by splitting my ego into participant and observer roles, I aim to respond antiphonally to the dual activity that characterized Freud's own compositional creativity ${ }^{56}$.

Peut-être pourrions-nous suggérer à notre tour que ce double mode discursif toujours en alternance et souvent mélangé au sein d'un même texte chez Freud nous semble surdéterminer chez ses traducteurs deux fantasmes, l'un tout de dogmatisme et de rigueur scientifique, l'autre tout à ses explorations génétiques dans le corps de la langue. De la note refusée comme épanchement narcissique à la glose envahissante, de la traduction comme science du sens à la traduction comme processus de transformation: il ne s'agit pas d'opter, de façon exclusive, pour l'une ou l'autre voie, mais plutôt de lire ce transfert à l'œuvre dans la traduction et de voir à quel point ces deux voies sont en réalité aussi bien inséparables qu'inévitables. Notant que Freud décrit lui-même trois types de lecteurs dans "Analyse avec fin et sans fin»: le sceptique, l'optimiste et l'ambitieux, Mahony ajoute dans sa conclusion, et ce sera également la nôtre ici, que

Of course, the different kinds of readers, like analysis, are unending. Nevertheless, the specificity of a psychoanalytic reading of Freud [j'ajouterai d'une traduction tout aussi bien] demands analysis of one's transference to him. And depending on the text, the reader [ou le traducteur] should analyse his transferences to Freud's patients as well to the constructions of incompetent and ideal reader that Freud himself proposes. These tasks lead us to realize that a genuine psychoanalytic reading, like analysis itself, is unending and ultimately indeterminate ${ }^{57}$.

Car traduire Freud, cela devrait aussi vouloir dire pour le sujettraducteur d'en passer par l'apprentissage d'une liberté qui serait, à

$\begin{array}{ll}\text { 56. } & \text { Ibid., p. } 94 . \\ \text { 57. } & \text { Ibid., p. } 95 .\end{array}$ 
sa manière et toutes proportions gardées, une forme d'expérience analytique, variable en profondeur et en qualité selon le rapport particulier de ce lecteur-traducteur, de celui-là et pas un autre, avec sa langue-mère et la langue (de l') autre. Traduire, ce peut toujours être cette opération par laquelle on cherche à rendre seulement le plus exactement possible le sens du texte, à imposer l'Univoque, à établir un rapport de maîtrise, d'autorité, de fidélité à la Tradition et à la transmission elle-même. Mais ce peut être aussi, ce devrait être un tout autre processus, grâce auquel toute une critique, une interprétation renouvelées du texte peuvent se mettre en place, dans une véritable ouverture dialogique attentive à l'équivoque, aux valeurs fluctuantes des métaphores et des différences qui seules peuvent donner accès non à la "vérité " du texte freudien, quelle qu'elle puisse être, mais à la justesse de son dire et de sa "libre parole».

Des métaphores de la traduction dont nous avons esquissé l'analyse ici, force est de constater que, de l'équipe Laplanche à Goldschmidt à Mahony, les affects qui viennent colorer les différentes positions théoriques varient chaque fois en fonction du transfert contracté par chaque traducteur individuel, mais également - et cet aspect mériterait à lui seul un long développement - en fonction de ce que, à défaut d'un meilleur terme, il faut désigner avec la prudence requise du nom de Sujet-Nation. En effet, il faut remarquer que le rapport à l'allemand comme langue (de l') autre n'est pas le même d'un espace socio-politique à l'autre et s'inscrit lui-même dans une tradition culturelle complexe, laquelle continue précisément de se transmettre au sens fort du terme, au gré de ses ouvertures et de ses résistances, par le biais de cette problématique de la traduction. Ce n'est certes pas la même chose de rendre «l'hindou, le grec, l'anglais en allemand plutôt que de rendre l'allemand par l'hindou, le grec, l'anglais ", comme le disait Pannwitz cité par Benjamin, et chaque langue entraîne peut-être, au delà ou en deça des sujets traducteurs particuliers qui s'y confrontent, une "épreuve de l'étranger » qui differe considérablement quant à la possibilité même de traduire dans une langue donnée. Jusqu'où ce Sujet-Nation propre à chaque langue influence-t-il notre manière de concevoir la tâche du traducteur, sa liberté et sa responsabilité théoriques et pratiques? S'il est difficile et sans doute prématuré de prendre la mesure d'une question aussi vaste à partir des exemples ponctuels qui nous retiennent ici, notons cependant pour finir que la première métaphore, celle défendue par l'équipe de Laplanche, est préoccupée par l'idée, toute légitime (et cherchant à imposer sa légitimité avec force), de restituer non pas tant le parcours parfois trouble des «mots à l'œuvre» de Freud, pour reprendre l'expression de Jean-Michel Rey, ou la malléabilité du matériau freudien, mais une interprétation unifiée, cohérente et surtout scientifique d'un texte freudien parfaitement accordé à une certaine institutionnalisation 
de la psychanalyse, alors que celui-ci ne cesse d'y faire des brèches lui-même, de l'intérieur même de son écriture. Seules les secondes images de la traduction du texte freudien, mises en pratique par Goldschmidt et Mahony mais aussi par d'autres traducteurs-critiques de l'œuvre freudienne (la nouvelle équipe qui retraduit la traduction de Strachey, l'équipe du Coq-Héron), engagent à notre avis différemment son avenir, en sautant le pas au-delà, en débordant programmes, protocoles et glossaires "raisonnés", en choisissant de lire les métaphores du texte freudien comme les lieux privilégiés, les passages nécessaires où s'inscrit déjà en creux tout un travail de traduction, linguistique et psychanalytique. À leur tour, ces métaphores de la traduction peuvent alors promettre - et cette promesse constitue, rappelons-le, la tâche la plus haute de la traduction dans son rapport à la culture - « une réconciliation possible entre les langues ${ }^{58}$ ": seule la traduction qui aura cette audace-là permettra dans l'après-coup à l'original de croître et de s'épanouir, selon le souhait de Benjamin.

Université de Montréal

58. Jacques Derrida, op. cit., p. 163. 